ISSN: 0212-5374

DOI: http://dx.doi.org/10.14201/et2015331193212

\title{
INTERVENCIÓN COMUNICACIONAL EN LA PROMOCIÓN DEL PERFIL AMBIENTAL DEL PROFESORADO DEL COLEGIO NACIONAL INTEGRADO JORGE BASADRE EN SANTA MARÍA DEL VALLE, HUÁNUCO. PERÚ-2011
}

Communicational intervention in promoting effective environmental profile of teachers of integrated National School Jorge Basadre in Santa María del Valle, Huánuco, Perú-2011

Intervention communicationnelle dans la promotion de profil environnemental des enseignants. Jorge Basadre Santa María del Valle, Huánuco, Peru-2011

\author{
A. SOTOMAYOR BACA \\ Correo-e: ansoba7@botmail.com
}

Recibido: 07-02-2014; Aceptado: 25-10-2014; Publicado: 30-04-2015 BIBLID [0212-5374 (2015) 33, 1; 193-212]

Ref. Bibl. A. SOTOMAYOR BACA. Intervención comunicacional en la promoción del perfil ambiental del profesorado del Colegio Nacional Integrado Jorge Basadre en Santa María del Valle, Huánuco. Perú-2011. Enseñanza \& Teaching, 33, 1-2015, 193-212.

RESUMEN: En la búsqueda de medidas de mitigación y remediación de la crisis ambiental, la preparación de profesores para la Educación Ambiental es, en 
A. SOTOMAYOR BACA

INTERVENCIÓN COMUNICACIONAL EN LA PROMOCIÓN...

la actualidad, reconocida como una prioridad. Ello determina la necesidad de una transformación continua del rol docente, constituyéndose en agentes activos en la formación integral de nuevas generaciones, abordando los problemas ambientales con enfoques emergentes.

El objetivo de esta investigación es comprobar si a lo largo de la intervención comunicacional se logra mejorar el perfil ambiental del profesorado del Colegio Nacional Integrado Jorge Basadre en Santa María del Valle, del distrito y provincia de Huánuco-Perú. Y, por tanto, optimizar sus capacidades hacia la enseñanzaaprendizaje del cuidado del ambiente, incorporando en el alumno el compromiso con el cuidado del ambiente. El estudio es experimental, prospectivo, longitudinal y analítico, con diseño de un solo grupo antes y después; de treinta y seis participantes voluntarios de la intervención comunicacional, desarrollada en sesiones mensuales, en un periodo de cinco meses. Se aplicaron satisfactoriamente una encuesta y dos escalas validadas y fiabilizadas. Se comprueban las hipótesis mediante el análisis de varianza de un factor de contrastes multivariados y la prueba t para medias de una muestra relacionada; apoyados en el SPWs V19. La influencia de la intervención comunicacional es demostrada mediante las diferencias significativas obtenidas en los momentos posteriores a la intervención respecto al momento basal. Según los hallazgos, se confirman las hipótesis que establecen diferencias en el comportamiento habitual, en la práctica pedagógica y en las percepciones hacia la educación ambiental de los participantes. Sin embargo, la intervención no mejora la práctica de valores y principios ambientales del profesorado, lo que supone la incidencia de futuros estudios que favorezcan tales cambios. De manera general, la intervención comunicacional aplicada es favorable en la promoción del perfil ambiental efectivo del profesorado en estudio.

Palabras clave: programa de formación docente; educación ambiental; eficacia de los maestros; comportamiento del profesor; escalas.

SUMMARY: In the search for mitigation and remediation of the environmental crisis, preparing teachers for Environmental Education is now recognized as a priority. This determines the need for a continuous transformation of the teaching role, becoming active agents in the formation of new generations, addressing environmental problems with emerging approaches.

The objective of this research is to check whether along the communicational intervention is possible to improve the environmental profile of teachers Jorge Basadre Integrated National College in Santa Maria Valley, the district and province of Huanuco, Peru. And, therefore, optimize their abilities to teaching-learning environment care, incorporating the student's commitment to protect the environment. The study was experimental, prospective, longitudinal, analytical, methodological with a single group before and after; volunteers participating on communication design based on a intervention, developed in monthly sessions in a five-month period thirtysix. A survey and two scales were validated and successfully applied. Hypotheses are tested by analysis of variance and multivariate contrasts stockings t-test shows a related; SPWS supported in V19. The influence of speech communication is demonstrated by the significant differences obtained in the moments after intervention from 
baseline. According to the findings, the hypotheses that differentiate in the normal behavior, teaching practice and perceptions towards environmental education participants are confirmed. However, the intervention did not improve the practice of environmental values and principles of teaching, which is the incidence of future studies that support such changes. In general, the communication intervention applied, is favorable in promoting effective environmental profile of teachers in the study.

Key words: teacher education program; environmental education; teacher effectiveness; teacher behavior scales.

RÉSUMÉ: Dans la recherche de l'atténuation et de l'assainissement de la crise environnementale, les enseignants se préparent pour l'éducation environnementale est maintenant reconnu comme une priorité. Ceci détermine la nécessité d'une transformation continue du rôle de l'enseignement, de devenir des agents actifs dans la formation des nouvelles générations, la résolution des problèmes environnementaux nouvelles approches.

L'objectif de cette recherche est de vérifier si le long de l'intervention de communication est possible d'améliorer le profil environnemental des enseignants Jorge Basadre Collège national intégré à Santa Maria Valley, le district et la province de Huanuco, Pérou. Et donc d'optimiser leurs capacités de soins de l'environnement d'enseignement et d' apprentissage, en intégrant l'engagement de l'élève à la protection de l'environnement. L'étude était expérimental, prospective, longitudinale, analyse, conception avec un seul groupe avant et après; volontaires participant intervention de communication, développé en sessions mensuelles sur une période de cinq mois trente-six. Une enquête et deux échelles ont été validées et fiabilizadas appliquées avec succès. Les hypothèses sont testées par analyse de variance et les contrastes multivariée bas T - test montre une est associée; SPWs supporté en V19. L'influence de la communication de la parole est démontrée par les différences significatives obtenues dans les moments après l'intervention de base. Selon les résultats, les hypothèses qui différencient dans le comportement normal, les pratiques pédagogiques et les perceptions envers les participants d'éducation environnementale sont confirmées. Cependant, l'intervention n'a pas amélioré la pratique des valeurs et principes de l'enseignement de l'environnement, qui est l'incidence de futures études qui prennent en charge ces changements. En général, l'intervention de communication appliquée, est favorable à la promotion de profil environnemental effective des enseignants dans l'étude.

Mots clés: programme des enseignants de l'enseignement; l'éducation environnementale; l'efficacité des enseignants; des échelles de comportement des enseignants. 
A. SOTOMAYOR BACA

INTERVENCIÓN COMUNICACIONAL EN LA PROMOCIÓN...

\section{INTRODUCCIÓN}

La grave problemática ambiental que se vivencia en los momentos actuales, caracterizada por la contaminación del agua, aire y suelo; la destrucción de la capa de ozono, que genera el cambio climático, entre otros problemas ambientales (Moreno, 2005), forman parte de lo cotidiano, habiéndose convertido en el foco de discusión social desde inicios del nuevo siglo (Sánchez, Tamayo, Barbería Fernández, Soler y Soto, 2011; Zeballos, 2005), en el análisis de la causalidad de la problemática ambiental, lo atribuye en primer orden a las pautas de comportamientos, actitudes, valores personales y al modo de vida nociva del ser humano, dado que el estilo de vida del mundo moderno está basado en el consumismo, la cultura del "usar y tirar", acumular indiscriminadamente los bienes y recursos naturales. En segundo orden de causas, considera a la ausencia de políticas de desarrollo por parte del Gobierno y la falta de liderazgo de las instituciones educativas, quienes son los responsables de la formación de los estudiantes.

En tal efecto, en la actualidad, se han formulado todo un conjunto de reformas educativas, que tratan de dar respuesta de las necesidades de este mundo globalizado. Todas ellas han proporcionado nuevas expectativas para favorecer la práctica de una cultura ambiental como resultado de la acción acertada del docente, cuyo papel es predominante en el alcance de competencias y habilidades en una formación integral de los individuos, para mejorar la calidad de vida (Sandoval, 2005). En consecuencia, amerita rescatar el rol activo y el compromiso de las funciones medulares de la educación ambiental, que se la concibe como una dimensión que debe ser atendida desde todos los sectores de aprendizaje, cuyo currículo proporcione objetivos y enfoques nuevos. Por tanto, las instituciones educativas y, específicamente, los responsables de la formación de los estudiantes requieren asimilar un papel o perfil relevante (hacia la enseñanza del cuidado y respeto al ambiente).

Sin embargo, la Organización de las Naciones Unidas para la Educación, la Ciencia y la Cultura (UNESCO) insta a considerar a la educación ambiental en el Diseño Curricular Nacional, pero esto no aparece como prioridad en los diferentes documentos de gestión curricular.

En Perú, el Ministerio de Educación incorporó en el Diseño Curricular de Educación Básica los ejes transversales: lenguaje, desarrollo del pensamiento, valores, ambiente y trabajo; sin embargo, no se concretiza una reforma curricular propiamente dicha, sino adaptaciones sucesivas, que han hecho aparecer contenidos ambientales en el nivel primario en asignaturas como, por ejemplo, la biología o ciencias, tecnología y ambiente. Los contenidos ambientales en secundaria están también en otras disciplinas, de forma muy general; esto ocurre en química de tercer grado o en biología del cuarto grado de secundaria. De este modo, la educación ambiental se protagoniza en teoría para exponerlo en el aula de clase y aprobar la materia. 
En la Región Huánuco, el perfeccionamiento docente enfatiza generalmente temas del quehacer educativo (metodología, didáctica etc.), siendo poco tratado la educación ambiental como conocimiento interdisciplinar por asignaturas. Del mismo modo, en las Instituciones Educativas del distrito de Santa María del Valle, de la provincia y departamento de Huánuco, se observa la poca relevancia de la educación ambiental, ya que el individuo continúa en su quehacer cotidiano con una conducta antiambientalista, lo que supone que no hubo aprendizaje; por tanto no tiene la iniciativa de emprender acciones conscientes en su medio familiar y comunal en defensa y conservación de su ambiente (Bers, 2011). Este hecho subraya la necesidad de la instrumentación de la educación ambiental, tanto en contenidos como en procedimientos didácticos para el cambio, constituyéndose en un espacio que cimiente la solución de la problemática ambiental.

Los cambios hacia la mejora cuantitativa y cualitativa hacen necesario el fortalecimiento del papel docente, compensando la debilidad de la formación inicial (López, 2011). Pues los docentes son los elementos fundamentales del proceso educativo, porque a través de su práctica docente tienen la posibilidad de incorporar orientaciones y propuestas que promuevan la reflexión y la sensibilización de los alumnos, dando pautas específicas para la acción. En este sentido, los docentes se constituyen en mediadores entre el conocimiento y el alumno, permitiendo la formación de una ética ambiental, que genere actitudes y nuevas formas de pensamiento hacia el cuidado ambiental.

En consecuencia, la presente investigación que aborda la formación del perfil ambiental del profesorado se basa en el modelo de la conducta ecológica responsable, que incluye todas aquellas actividades de los seres humanos cuyo énfasis es la reducción del deterioro ambiental (Corral, 2001; Grob, 2005). El estudio centra su interés en la aplicación de intervenciones mediante estrategias de enseñanza aprendizaje hacia una visión integral de la problemática ambiental y su complejidad, a fin de generar conocimiento hacia comportamientos proambientales en el profesorado del Colegio Nacional Integrado Jorge Basadre en Santa María del Valle, de la provincia y departamento de Huánuco, durante el 2011.

También, guio la presente investigación el enfoque teórico del comportamiento planeado, en la teoría de la transversalidad de la educación ambiental, en la promoción de la salud en la orientación de la protección ambiental, la participación comunitaria, la teoría de acción ambiental positiva de Emmons, la teoría sistémica del medio ambiente y el modelo de cambio individual de conducta o modelo transteórico entre otras; todas ellas, en su conjunto, proporcionan un referente teórico para explicar la intervención comunicacional hacia la formación del perfil ambiental efectivo del docente. 
A. SOTOMAYOR BACA

INTERVENCIÓN COMUNICACIONAL EN LA PROMOCIÓN...

2. LA INTERVENCIÓN COMUNICACIONAL COMO ESTRATEGIA DE FORMACIÓN DEL PERFIL AMBIENTAL DEL DOCENTE Y PROMOTOR AMBIENTAL

Las intervenciones comunicacionales constituyen un conjunto de procedimientos y acciones innovadores encaminados a la difusión de conocimientos, desde las demandas del cuidado ambiental, basadas en una nueva concepción de cultura socioambiental. Utilizan estrategias de difusión que tienden a lograr cambios en los conocimientos, actitudes y comportamientos de los docentes, motivándolos hacia la participación de acciones concretas que contribuyan a la solución de la problemática ambiental (Morán, 2014); para asegurar el bienestar de la población, con un enfoque hacia el desarrollo sostenible (Sánchez, 2011; Bers, 2011) de los procesos económicos, sociales y culturales.

Según Chocano (2011), las intervenciones comunicacionales promueven las experiencias de aprendizaje para relevar la importancia del ambiente en la vida y la realidad de las personas, y están básicamente orientadas a un cambio de actitudes en relación con la protección ambiental. Esto, como un proceso de sensibilización del respeto, valor y cuidado de la vida, para evitar el deterioro del ecosistema y aminorar el impacto ambiental negativo. Mediante ello, los docentes participan involucrándose y garantizando que los escolares tengan el acceso al derecho de recibir un mensaje veraz, oportuno y adecuado a través de actividades de información, educación y comunicación para promover la participación, tendiente a generar un compromiso solidario y responsabilidad compartida en el cuidado del ambiente.

\section{EL PERFIL DEL DOCENTE EN EDUCACIÓN AMBIENTAL}

El perfil del docente en Educación Ambiental supedita el dominio de un conjunto de conceptos y teorías que le permitan aplicar los procesos didácticos a fin de que los alumnos interpreten su medio ambiente en toda su complejidad. Debe crear las condiciones óptimas para que se produzca una interacción constructiva entre al alumno y el objeto de conocimiento. Debe desarrollar habilidades que le permitan investigar, evaluar y participar activamente en la prevención de los problemas ambientales y ser un facilitador activo en todo momento del proceso educativo sobre el cuidado del medio ambiente. Finalmente, debe ejercer la educación para la responsabilidad ambiental y para el desarrollo armonioso de las relaciones intra- e intersociedades (educación para el mundo).

Entre los antecedentes de esta investigación destacan los estudios de Acebal (2010), que en España ejecutó un estudio cualitativo, titulado "Conciencia Ambiental y Formación de Maestros", cuyo objetivo fue conocer mediante una encuesta la conciencia ambiental de los docentes, cuya población estuvo conformada por 155 estudiantes.

En México, Camarena (2009) realizó un estudio descriptivo, prospectivo, comparativo y transversal, titulado "EA y formación del profesorado: estudio de perfiles ambientales en las escuelas normales de Sonora", cuyo objetivo fue identificar en el 
INTERVENCIÓN COMUNICACIONAL EN LA PROMOCIÓN...

imaginario social de docentes y estudiantes los atributos que presentan sus respectivos perfiles ambientales, con el fin de ofrecer una aproximación explicativa de la escala social de valores en la mejora del ejercicio de la EA formal y el perfil ambiental del docente. Y en España, Vega (2004) abordó un estudio cuasiexperimental, sobre la educación ambiental en la formación inicial del profesorado basado en un análisis de un modelo didáctico para el desarrollo de la competencia para la acción a favor del ambiente, cuyo objetivo fue determinar una metodología didáctica para la formación inicial del profesorado en educación ambiental y comprobar si los alumnos, futuros profesores, siguen esta metodología, si obtienen mejores resultados en los distintos campos del aprendizaje (estructuras conceptuales, aptitudes, actitudes y comportamiento) en relación con el entorno.

\section{MATERIAL Y MÉTODOS}

\subsection{Planteamiento de la investigación}

Por las justificaciones señaladas, surge la necesidad de una redefinición del perfil ambiental de los docentes, que esté caracterizada por una transformación continua, con procesos de construcción y reconstrucción permanente de la educación ambiental, razón por la cual se realiza la investigación con el objetivo principal de comprobar si la aplicación de la intervención comunicacional y a lo largo de la misma se consigue mejorar el perfil ambiental del profesorado del Colegio Nacional Integrado Jorge Basadre de Santa María del Valle, del distrito y provincia de Huánuco-Perú; asumiendo que cuanto mayor sea el número de docentes con conocimientos, valores, comportamientos ambientales adecuados y que, a su vez, estén dispuestos a incluirlos en su propia vida y en su quehacer docente, más cerca estaremos al logro de los objetivos de la educación ambiental.

El propósito es analizar si la intervención comunicacional favorece a los docentes en la optimización de sus capacidades hacia la enseñanza-aprendizaje del cuidado del ambiente, incorporando en el estudiante una visión humanista del compromiso con el cuidado del ambiente.

\subsection{Metodología}

Se efectuó un estudio de tipo cuasiexperimental, prospectivo, longitudinal y analítico que se basa en el análisis de datos cuantitativos, metodología utilizada para cuantificar fenómenos y poder establecer relaciones y explicaciones causales para generar, justificar y comparar hipótesis. El diseño de investigación fue de un solo grupo antes y después, con cuatro mediciones durante el estudio: antes, posintervención, a tres y seis meses después de la intervención.

Los datos pertenecen a una muestra no probabilística por conveniencia, conformada por la totalidad (36) de los profesores del colegio en estudio (población muestral), según los criterios de inclusión y exclusión. Alrededor de la mitad de ellos fueron del sexo femenino (55,6\% [20]) y de estado civil soltero/a (52,8\% [19]). 
La gran mayoría refirieron profesar la religión católica (83,3\% [30]) y el grupo etario preponderante fue de 40 a 60 años (72,2\% [26]). Entre las características laborales, se observó que la mayoría tuvieron la condición de nombrados (83,3\% [30]); con un tiempo laboral mayor a 11 años (72,2\% [26]) indicando amplia experiencia docente; y con grado académico de licenciados en educación (63,9\% [23]). Y, en cuanto a la participación en aspectos ambientales, la mayoría (86,1\% [31]) refirió que, alguna vez, participaron en actividades de educación ambiental. Del mismo modo, recibieron información sobre aspectos ambientales durante su formación profesional (86,1\% [31]) para ambos]; y actualmente recibieron información ambiental una proporción considerable de ellos (66,7\% [24]).

Los instrumentos de recolección de datos fueron: una encuesta de características sociodemográficas, laborales y de información ambiental; las escalas de valores y principios en educación ambiental; de comportamientos habituales en educación ambiental; de percepciones de la educación ambiental y de prácticas pedagógicas en la educación ambiental; dichas escalas fueron validadas a distintos niveles y en dos fases, una cualitativa, correspondiente a la creación de dichos instrumentos (validez cualitativa de contenido para todos los instrumentos de medición), realizada a través de la apreciación de 11 jueces (expertos en investigación, experiencia de trabajo con docentes, del mismo modo docentes catedráticos con gran experiencia en educación secundaria, docentes expertos en educación ambiental, etc.); los mismos que recibieron suficiente información escrita acerca del propósito del estudio; objetivos e hipótesis, operacionalización de las variables, y los instrumentos de recolección de datos. Los jueces dieron a conocer sus distintos puntos de vista, hubo observaciones salvables, las mismas que fueron revisadas, reformuladas, y nuevamente validadas tres días después del acontecimiento, asimismo, quedaron excluidos algunos ítems donde no hubo coincidencia de los expertos; finalmente todos los jueces consideraron pertinente su aplicación a la muestra en estudio.

Además, se evaluaron las propiedades métricas (confiabilidad), por el método Alpha de Crombach [Escala de valores y principios en educación ambiental $(\alpha=0,623)$, escala de comportamientos habituales en educación ambiental $(\alpha=0,721)$, escala de percepciones sobre la educación ambiental $(\alpha=0,735)$, escala de prácticas pedagógica en la educación ambiental $(\alpha=0,748)$; previo a su aplicación por tres encuestadores capacitados.

En el análisis inferencial de los resultados, inicialmente se realizó la prueba de normalidad, aplicando el estadístico de Kolmogorov-Smirnov, en las distribuciones que contrastan las hipótesis. En segundo lugar, se emplearon el Análisis de Varianza (ANOVA) y una prueba de comparación de medias (prueba t para una muestra, teniendo en consideración las variables cuantitativas), en la cual se consideró un valor $\mathrm{p} \leq$ de 0,05; apoyados en el PASW versión 19.0 para Windows.

La intervención comunicacional se basó en el modelo de información, educación y comunicación (IEC), el mismo que incluyó la propuesta metodológica de 
entornos de aprendizaje constructivista que propició la reflexión crítica y apropiación del conocimiento en materia de cuidado ambiental (Nieda Juana y Macedo, 1997).

El proceso de capacitación siguió una metodología de aprendizaje en la convivencia, la participación, la reflexión crítica y el desarrollo de capacidades orientadas a socializar el conocimiento. Se trata de un proceso inductivo que, partiendo de la reflexión individual y colectiva sobre experiencias personales y del entorno, promueve un análisis crítico desde los enfoques de sostenibilidad ambiental. Estos procesos formativos se apoyaron tanto o más que en nuevos conocimientos, en las vivencias personales que facilitan la apropiación de contenidos, desde la experiencia misma de los participantes. El taller se organizó alrededor de tres funciones básicas: motivadora, informativa y productiva. En general, las primeras sesiones tuvieron la función motivadora, mientras que las centrales llevaban la mayor carga informativa. Las últimas sesiones se centraron en los aspectos productivos del taller (distribución de material educativo: paneles informativos o murales con información sobre el cuidado del ambiente, dípticos, trípticos, volantes, folletos, otros).

La frecuencia de las sesiones fue mensual (en un periodo de cinco meses). Se asignó un colaborador para cada sesión, junto a la investigadora, que estuvo presente en todas las sesiones educativas. La intervención aplicada fue inocua, considerándose los principios éticos, como el consentimiento informado, por tanto, su método le confiere eficacia en la promoción del perfil ambiental efectivo del profesorado.

\section{RESUltados}

Inicialmente se analizaron las réplicas de la variable práctica de valores y principios ambientales en educación ambiental en el profesorado en estudio, evidenciándose que la intervención comunicacional no mejoró dichas prácticas. Ello supone que existen vacíos en los comportamientos específicos y pertinentes que contribuyen al perfil ambiental de los docentes, estando debilitados el valor de la libertad, el sentido de igualdad y de justicia, de cuidar de los débiles, el respeto a la tierra, la protección del medio ambiente, la evitación de la contaminación ambiental y la proyección del bienestar de las futuras generaciones, entre otros. Este hecho se confirma dado que las tres medias posteriores a la intervención (después $=59,1 ;$ a 3 meses $=59,0$ y a 6 meses $=59,0)$ fueron similares a la del momento inicial $(59,5)$. Al contrastar estos valores mediante el análisis de varianza con un factor, no hubo diferencias significativas en los 4 momentos del estudio, según el estadístico de diferencia de contrastes multivariados cuyo valor fue $F=1,1$ y $\mathrm{p}=$ 0,367. En base a ello, no se pudo rechazar la hipótesis nula de igualdad entre las réplicas.

En segundo, tercer y cuarto análisis, se evaluaron las réplicas de las variables comportamiento habitual, percepciones y práctica pedagógica en educación 
A. SOTOMAYOR BACA

INTERVENCIÓN COMUNICACIONAL EN LA PROMOCIÓN...

ambiental del profesorado en estudio, las que evidenciaron que la intervención comunicacional incrementa los valores promedios de dichas variables.

En base a estos hallazgos, se rechazaron las hipótesis nulas, admitiéndose que el comportamiento habitual, las percepciones y prácticas pedagógicas en educación ambiental del profesorado en estudio fueron diferentes durante los cuatro momentos del estudio indicando que la intervención comunicacional incrementa los valores promedios de dichas variables.

Al referirse al comportamiento habitual expresado como favorable, pone de manifiesto que los docentes, como resultado de la intervención comunicacional, adoptan una vida equilibrada entre trabajo y la vida privada, se mantienen en contacto con la naturaleza, colaboran en tareas de reforestación y conservación de áreas verdes, viven de forma sencilla y consumen solo lo necesario. Asimismo, asuman un rol activo en defensa del medio ambiente, informándose sobre los principales problemas y daños al ambiente (global y local), ayudando a prevenir la destrucción de la capa de ozono y del medio ambiente natural, de la acumulación de sustancias contaminantes, y el cambio climático, cambiando su estilo de vida y sus conductas diarias en beneficio del medio ambiente, y ahorrando recursos y energía, entre otros.

Y, respecto a las percepciones a favor de la educación ambiental, los docentes muestran interés por la protección ambiental, mediante la participación de las instituciones educativas. Asumen posturas críticas y éticas en torno a conflictos ecológicos. Integran a diversas disciplinas e instituciones y sectores en las acciones del cuidado ambiental, mantienen vigentes los conceptos de desarrollo sostenible, degradación del planeta, calidad de vida, entre otros.

Y en la dimensión práctica pedagógicas, supone que el docente brinda al estudiante de manera sistemática información sobre la problemática ambiental contemporánea y cómo ésta se manifiesta en nuestra comunidad (problemáticas locales), sobre distintas propuestas y enfoques de educación ambiental. Tiene iniciativa por promover la educación ambiental enfatizando el medio ambiente natural. Presenta contenidos referidos a la interacción del hombre con su entorno global, propicia que docentes y estudiantes organicen y emprendan acciones a favor del medio ambiente. La temática ambiental se aborda de manera transversal en varias materias, entre otros.

Esto fue evidenciado en la medición de las tres medias posteriores a la intervención, que fueron superiores a la media inicial; y que al aplicar el análisis de varianza para un factor de las medidas repetidas, durante los 3 momentos posteriores, mostraron mediante el estadístico de comparaciones multivariadas cambios significativos cuyos valores fueron: $\mathrm{F}=46,0$ y $\mathrm{p}=0,000$ para la variable comportamiento habitual hacia la educación ambiental; $\mathrm{F}=69,3$ y $\mathrm{p}=0,000$ para la variable percepciones hacia la educación ambiental; y $\mathrm{F}=46,0$ y $\mathrm{p}=0,000$ para la variable práctica pedagógica en educación ambiental.

Mediante la prueba t de una muestra relacionada, para hipótesis a una sola cola, se compararon los promedios de los tres momentos posteriores a la 
intervención de las variables: comportamiento habitual hacia la educación ambiental: $(77,9)$ Vs $(67,67)$, observándose una diferencia de 10,2 puntos (IC 95\% $=7,8-12,5)$, siendo significativa $[\mathrm{t}=8,8 ; \mathrm{p}=0,000]$, ver Tabla 4; percepciones en educación ambiental: $(126,6)$ Vs $(103,53)$, cuya diferencia fue de 23,1 puntos [(IC $95 \%$ de 7,8 a 12,5) siendo significativa $\mathrm{t}=26,9$ y $\mathrm{p}=0,000$ ], ver Tabla 2; y prácticas pedagógicas en educación ambiental: $(73,0)$ Vs $(58,25)$, cuya diferencia fue de 14,7 puntos (IC 95\% de 12,8 a 16,7); evidenciándose significancia estadística [t = 15,2; p $=0,000$, ver Tabla 3; con estos resultados se rechazó la hipótesis nula y se admite que el promedio de los tres momentos posteriores a la intervención fue superior al del momento inicial. De este modo, se admite que la intervención comunicacional en el profesorado en estudio mejoró el comportamiento habitual hacia la educación ambiental (ver Tabla 1), las percepciones en educación ambiental (ver Tabla 2) y la práctica pedagógica en educación ambiental (ver Tabla 3).

TABLA 1

Prueba t de comparación de medias de una muestra entre el valor basal y los tres momentos posteriores a la intervención, de la variable comportamiento habitual en educación ambiental por el profesorado del Colegio Nacional Integrado Jorge Basadre en Santa María del Valle, Huánuco-2011

\begin{tabular}{|c|c|c|c|c|}
\hline \multirow{2}{*}{$\begin{array}{l}\text { Prueba t para una muestra, comparación de } \\
\text { medias (pre- y posintervención) de la variable } \\
\text { comportamiento habitual en educación ambiental }\end{array}$} & \multicolumn{4}{|c|}{ VALOR DE PRUEBA $=67,67$} \\
\hline & $\mathrm{t}$ & p-valor & $\begin{array}{l}\text { Diferencia } \\
\text { de medias }\end{array}$ & IC $95 \%$ \\
\hline $\begin{array}{l}\text { Promedio de los comportamientos habituales ( } 3 \\
\text { momentos posteriores a la intervención respecto a } \\
\text { la pre intervención) }\end{array}$ & 8,8 & 0,000 & 10,2 & $(7,8-12,5)$ \\
\hline
\end{tabular}

Fuente: Escala de comportamientos habituales en educación ambiental.

TABLA 2

Prueba t de comparación de medias para una muestra entre: el valor basal

y los tres momentos posteriores a la intervención, de la variable percepciones hacia la educación ambiental por el profesorado del Colegio Nacional Integrado Jorge Basadre en Santa María del Valle, Huánuco-2011

\begin{tabular}{|l|c|c|c|c|}
\hline \multirow{2}{*}{$\begin{array}{l}\text { Prueba t para una muestra de la variable } \\
\text { percepciones hacia la educación ambiental }\end{array}$} & \multicolumn{4}{|c|}{ VALOR DE PRUEBA $=103,53$} \\
\cline { 2 - 6 } & $\mathrm{t}$ & $\mathrm{p}$-valor & $\begin{array}{c}\text { Diferencia } \\
\text { de medias }\end{array}$ & IC 95\% \\
\hline $\begin{array}{l}\text { Promedio de las percepciones (en los 3 } \\
\text { momentos posteriores a la intervención) }\end{array}$ & 26,9 & 0,000 & 23,1 & $(21,3-24,8)$ \\
\hline
\end{tabular}

Fuente: Escala de percepciones en educación ambiental. 
TABLA 3

Prueba t para comparación de medias de una muestra entre el valor basal y los tres momentos posteriores a la intervención, de la variable práctica pedagógica en educación ambiental por el profesorado del Colegio Nacional Integrado Jorge Basadre en Santa María del Valle, Huánuco-2011

\begin{tabular}{|l|c|c|c|c|}
\hline Prueba t de comparación de medias para una & \multicolumn{4}{|c|}{ VALOR DE PRUEBA $=58,25$} \\
\cline { 4 - 6 } $\begin{array}{l}\text { muestra de la variable práctica pedagógica en } \\
\text { educación ambiental }\end{array}$ & $\mathrm{t}$ & $\mathrm{p}$-valor & $\begin{array}{c}\text { Diferencia } \\
\text { de medias }\end{array}$ & IC $95 \%$ \\
\hline $\begin{array}{l}\text { Promedio de la variable práctica pedagógica } \\
\text { momentos posteriores a la intervención) }\end{array}$ & 15,2 & 0,000 & 14,7 & $(12,8-16,7)$ \\
\hline
\end{tabular}

Fuente: Escala de práctica pedagógica en educación ambiental.

De forma general, se compararon las medias de las réplicas de la variable perfil ambiental del profesorado en estudio, en el que se evidenció que las tres medias posteriores a la intervención (después $=318,8$; a 3 meses $=319,7$, y a 6 meses $=321,8)$ fueron superiores al momento inicial $(277,9)$. Al realizar el análisis de varianza para un factor de las medidas repetidas durante los 4 momentos (antes $=277,9$; después $=318,8$; a 3 meses $=319,7$; y a 6 meses después $=321,8$ ), se observaron cambios. Ello fue estimado mediante el estadístico de diferencia $\mathrm{F}=$ 55,2 de contrastes multivariados, cuyo resultado fue significativo $(p=0,000)$. En consecuencia, se rechazó la hipótesis nula y se admitió que el perfil ambiental del profesorado del Colegio Nacional Integrado Jorge Basadre en Santa María del Valle, durante el 2011, fue diferente durante los cuatro momentos del estudio.

Finalmente, se aplicó la prueba t para una muestra con la hipótesis a una sola cola, donde se comparó el promedio de los tres momentos posteriores a la intervención $(320,0)$ con el valor del momento inicial $(227,92)$; evidenciándose diferencias de 42,2 puntos (IC $95 \%$ de 34,0 a 50,4 ) con significancia estadística [ $\mathrm{t}=10,4$; $\mathrm{p}=0,000]$, por lo que se rechazó la hipótesis nula, afirmándose que el promedio de los tres momentos posteriores a la intervención es superior al momento inicial. (Ver Tabla 4). Con este resultado se consideró que la intervención comunicacional, incrementó los valores promedios del perfil ambiental del docente.

\section{TABLA 4}

Prueba t de comparación de medias de una muestra entre el valor basal y los tres momentos posteriores a la intervención, de la variable perfil ambiental del profesorado del Colegio Nacional Integrado Jorge Basadre en Santa María del Valle, Huánuco-2011

\begin{tabular}{|l|c|c|c|c|}
\hline \multirow{2}{*}{$\begin{array}{l}\text { Prueba t para una muestra de la variable perfil } \\
\text { ambiental del profesorado }\end{array}$} & \multicolumn{4}{|c|}{ VALOR DEUEBA $=227,92$} \\
\cline { 2 - 5 } & $\mathrm{t}$ & $\mathrm{p}$-valor & $\begin{array}{c}\text { Diferencia } \\
\text { de medias }\end{array}$ & IC $95 \%$ \\
\hline $\begin{array}{l}\text { Promedio de perfil ambiental (3 momentos } \\
\text { posteriores a la intervención) }\end{array}$ & 10,4 & 0,000 & 42,2 & $(34,0-50,4)$ \\
\hline
\end{tabular}

Fuente: Escala de valores y principios en educación ambiental, de comportamientos habituales en educación ambiental, de percepciones sobre la educación ambiental, de prácticas pedagógica en la educación ambiental. 
INTERVENCIÓN COMUNICACIONAL EN LA PROMOCIÓN...

Con las hipótesis probadas se afirma que la intervención comunicacional aplicada mejora el perfil ambiental del profesorado del Colegio Nacional Integrado Jorge Basadre en Santa María del Valle, durante el 2011, confirmándose su efectividad.

\section{DiSCUSIÓN Y CONCLUSIONES}

Existe evidencia científica de que la cultura ambiental de los profesores influye en qué y cómo enseñan la educación ambiental (Hsu, González, Martín et al. en Corral, 2001), tal como afirma Terrón (2012), el profesor que reciba una formación ambiental sólida tendrá una visión sistémica de la problemática ambiental y podrá llevar adelante su tarea como educador ambiental, ya sea en el ámbito escolar o comunitario en el cual se desenvuelve, donde ha de tratar como tema transversal, con muchas garantías de éxito.

Los resultados obtenidos a partir de esta investigación sugieren que la intervención comunicacional aplicada fue efectiva, ya que mejoró el perfil ambiental del profesorado del Colegio Nacional Integrado Jorge Basadre en Santa María del Valle, durante el 2011. El efecto favorable de la intervención comunicacional se atribuye al efecto de las estrategias desarrolladas tales como las sesiones y diferentes actividades de aprendizaje que se fueron introduciendo de forma progresiva. Todo ello fue resultado de los aprendizajes significativos e integrales sobre el desarrollo de una ciudadanía y cultura ambiental responsable, sostenible, con enfoque de equidad, participativa, organizada, y con perspectivas de un encuentro entre escuela y comunidad. Se enfatizó también la promoción de estilos de vida saludables, la seguridad, la prevención y el uso eficiente y responsable de los recursos y de la diversidad natural, social y cultural; la relación sociedad-cultura-entorno, ambiente y desarrollo sostenible.

Este resultado va en la línea de Álvarez y Vega (2009), quienes plantean que los individuos solo realizan conductas ambientalmente responsables cuando están suficientemente informados sobre la problemática ambiental, se encuentran motivados hacia ella y se ven capaces de generar cambios cualitativos. Esto al estar convencidos de la efectividad de su acción y de que esta no les generará dificultades importantes.

Del mismo modo, este resultado va en consonancia con los modelos que predicen la realización de conductas responsables con el ambiente, propuestos por Corral Verdugo (2001), las que, a pesar de presentar algunas discrepancias entre ellos, coinciden en señalar que las actitudes y la intención de actuar tienen una importante influencia sobre el comportamiento cuando otros factores no impiden que este se lleve a cabo, sobre todo en lo referente a los comportamientos individuales de consumo y de participación ambiental. En este último, Sandoval (2011) destaca el papel del docente de educación básica como agente de cambio, ya que tiene un rol activo en la formación de nuevas generaciones y que a partir de su intervención exista una nueva oportunidad de construcción de alternativas sostenibles en el futuro del planeta. 
Este resultado responde a la perspectiva ecológica, que sostiene que los comportamientos tienen dos características fundamentales: son influenciables en múltiples niveles (por factores individuales, interpersonales, institucionales u organizativos, comunitarios y de políticas públicas) y son de causalidad recíproca con su entorno (Organización Panamericana de la Salud, 2011).

Además, este resultado supone que el docente, durante el proceso de enseñanza aprendizaje, procurará personas responsables y comprometidas con el cuidado ambiental. Hecho que se apoya en el modelo de cambio individual de conducta o modelo transteórico propuesto por Prochaska (1979), quien asume que el docente informado adecuadamente en materia de cuidado ambiental contará con conocimiento y desarrollará actitudes favorables frente a la protección ambiental, además, asumirá un rol activo frente a la educación ambiental.

El presente resultado también se explica con la postura utilitarista de la teoría de acción razonada de Fishbein interpretada por Pasco, Villafuerte y Neyra (2010), respecto a los procesos cognitivos de expectativa y utilidad subjetiva esperada donde la persona o la población adulta, al decidirse por una conducta relativa al ambiente, evalúa las consecuencias subjetivas esperadas de las diferentes conductas posibles y elige aquella que menos costos le supone y que más beneficios le aporta, independientemente del nivel de conocimiento ambiental. Esta teoría permite interpretar los factores determinantes para el aprendizaje de una actividad o de una conducta preventiva (Fishbein en Ríos Hernández, 2011).

El presente resultado coincide con las experiencias desarrolladas por la Secretaría de Educación Pública de México (2009), habiendo logrado éxito un programa para la Educación Secundaria, cuyo propósito fue que los docentes tengan la oportunidad de redimensionar su modo de actuar en un contexto donde se privilegie la indagación de los problemas que enfrenta el ambiente local, municipal y estatal, convirtiéndose en un facilitador participativo en la construcción y reconstrucción de la realidad. Asimismo, Morelos y Terrón (2011) han realizado estudios de intervención enfatizando al docente como promotor de la conservación y preservación del medio natural inmediato a partir del reconocimiento del eje transversal en la escuela secundaria.

Contrariamente con el presente hallazgo, Maldonado (2005) ha referido, basado en Hsu y Roth, Summer, Corney, Childs, Christenson, Ernst, que la educación ambiental no garantiza un comportamiento individual más responsable con la tierra. Plantea que en cierto modo es necesaria para lograr un mejor conocimiento y comprensión de los sistemas de soporte de la vida y que, por lo menos, aumenta la probabilidad de transitar hacia la sustentabilidad.

De otro lado la intervención comunicacional mejora las percepciones hacia la educación ambiental, las que podrían estar sujetas a las actividades cognoscitivas aplicadas en la intervención comunicacional sobre educación ambiental. Hecho que concuerda con la teoría cognitiva, que explica que es a través del cognitivismo por el que se puede estudiar cómo las personas perciben varias formas, por qué recuerdan algunas cosas y otras no, cómo se aprende el lenguaje o cómo piensan cuando tienen que resolver un problema (Sternberg en Ríos, 2011). 
La mejora de las percepciones hacia la educación ambiental posibilitó que los docentes tuvieran opiniones o creencias orientadas principalmente hacia la realización de actividades de formación en educación ambiental, adaptadas a los intereses del alumnado y de la forma más adecuada que permita la generación de conocimientos, valores y actitudes sobre determinados aspectos de su realidad ambiental inmediata. A su vez, actúan como modificadores tales: situaciones conflictivas de los espacios naturales, desarrollo sostenible, evitación de la degradación del planeta, calidad de vida, entre otros.

En lo que respecta a las percepciones favorables hacia la educación ambiental de los docentes, inducirá el pensamiento ético que orienta la acción humana en la naturaleza, regulándola mediante el establecimiento de derechos y obligaciones. En este caso, tal como lo señala Bellver (1997), los docentes pondrán énfasis en la preservación del sistema ecológico que lleva a una defensa férrea de la diversidad como fuente de estabilidad y desarrollo futuro. Este aspecto ha sido analizado por Fernández, Porter y Sureda (2010), ya que consideran necesario disponer de información rigurosa para la percepción social de los temas que configuran la problemática ambiental.

En el mismo sentido, las percepciones estarán orientadas a acciones mucho más sostenibles con el ambiente, que, según McLaughil (1999), las posturas de cambio en torno al ambiente pasarán desde los paradigmas antropocentristas y biocentristas a los ecocentristas. Es decir, se logrará modificar el extremo de la postura meramente utilitarista en la que el ser humano es el ser supremo del planeta, hasta asignar un valor intrínseco a la naturaleza, incluyendo a las cosas y procesos no vivos, sin tener en consideración especial al uso que el ser humano le ofrezca (pondera el predominio humano sobre la naturaleza).

Se podría argumentar también que la intervención comunicacional mejora la práctica pedagógica que imparten los docentes en educación ambiental, lo que posibilitará que se constituyan en modelos concretos, para que los estudiantes aprendan el cuidado ambiental, pues, según Parra (2003), es en el contexto escolar donde se encuentran los modelos concretos de aprendizaje, que, en el caso del presente estudio, los docentes que muestran coherencia entre su decir y su hacer representan modelos de comportamientos y prácticas a favor del ambiente, lo cual les concede más fuerza ante el alumno, que actúa como observador. Del mismo modo, la propia organización interna del aula favorece los procesos de imitación en el medio escolar, al darse en un contexto en el que hay numerosos modelos que hacen lo mismo.

Además, su práctica pedagógica será realizada con el enfoque de la interdisciplinariedad y la transversalidad, en la que los aprendizajes deben estar incluidos en todas las materias a fin de potenciar en los estudiantes actitudes y conductas que ayuden a desarrollar su capacidad reflexiva, su autoestima, su sentido de pertenencia; incentivarlos a trabajar por una buena convivencia escolar y familiar, promoviendo el respeto por el otro y por las formas de vida del planeta, y también brindarles las herramientas necesarias para desenvolverse en un mundo en permanente cambio. 
A. SOTOMAYOR BACA

INTERVENCIÓN COMUNICACIONAL EN LA PROMOCIÓN...

Las conclusiones de este estudio, que contribuye a evidenciar la influencia positiva de la intervención comunicacional de la formación del perfil ambiental efectivo del docente, son:

- $\quad$ Los promedios de la variable práctica de valores y principios ambientales en educación ambiental fueron similares durante los cuatro momentos del estudio cuyo valor estadístico de diferencia fue $\mathrm{F}=1,1$ y $\mathrm{p}=0,367$, no siendo significativo. En consecuencia, se aceptó la hipótesis nula referida a que la intervención comunicacional no mejora la práctica de valores y principios ambientales en educación ambiental del profesorado en estudio.

- Los promedios de la variable comportamiento habitual hacia la educación ambiental demostraron cambios significativos, durante los 4 momentos, que fue contrastado con el análisis de varianza de un factor de contrastes multivariados $(\mathrm{F}=46,0$ y valor $\mathrm{p}=0,000)$, por lo que se aceptó la hipótesis de diferencia; afirmando que el comportamiento habitual hacia la educación ambiental de los docentes en estudio fue diferente durante los cuatro momentos del estudio. Además, se comprobó que la intervención comunicacional mejora el comportamiento habitual en educación ambiental del profesorado en estudio.

- La variable percepciones hacia la educación ambiental en los momentos posteriores a la intervención demostró cambios significativos respecto a la media inicial $=103,5$, la que fue comprobada mediante el análisis de varianza para un factor de contrastes multivariados (valor del estadístico de diferencia $\mathrm{F}=69,3$, y $\mathrm{p}=0,000$ ). Con este resultado se rechazó la hipótesis nula y se aceptó que las percepciones hacia la educación ambiental de los docentes en estudio fueron diferentes durante los cuatro momentos del estudio.

Dado que el promedio de los tres momentos posteriores a la intervención fue superior al del momento inicial $[\mathrm{t}=26,9 ; \mathrm{p}=0,000]$, se rechazó la segunda hipótesis nula. A partir de estas dos afirmaciones se concluye que la intervención comunicativa mejora las percepciones que tienen los docentes hacia la educación ambiental.

- En el análisis de la variable práctica pedagógica en educación ambiental en los momentos posteriores a la intervención se observó superioridad significativa a la media inicial, esto fue comprobado con el análisis de varianza de un factor de contrastes multivariados (Valor estadístico de diferencia $\mathrm{F}=46,0$ y $\mathrm{p}=0,000)$. En consecuencia se rechazó la hipótesis nula y se aceptó que la práctica pedagógica en educación ambiental fue diferente durante los cuatro momentos del estudio. Posterior a ello, mediante la prueba t para la comparación de medias de una muestra con la hipótesis a una sola cola, se compararon los promedios de los tres momentos posteriores a la intervención con la del momento inicial $(58,25)$; evidenciándose diferencias significativas $[\mathrm{t}=15,2 ; \mathrm{p}=0,000]$, con lo que se rechazó la segunda hipótesis nula y se afirma que el promedio de los 
A. SOTOMAYOR BACA

INTERVENCIÓN COMUNICACIONAL EN LA PROMOCIÓN...

tres momentos posteriores a la intervención fue mayor al momento inicial. Esta diferencia fue de 14,7 puntos con un intervalo de confianza de 12,816,7. Con las dos hipótesis probadas se afirma que la intervención comunicativa aplicada mejoró la práctica pedagógica en educación ambiental que imparten los docentes.

- De manera general, se analizaron las medias para las réplicas de la variable perfil ambiental, en el cual se evidenció que las tres medias posteriores a la intervención (después $=318,8$; a 3 meses $=319,7$; y a 6 meses $=$ $321,8)$ fueron numéricamente superiores a la media inicial $(277,9)$. Este resultado se comprobó con el análisis de varianza para un factor de contrastes multivariados (valor estadístico de diferencia $F=55,2$ y $p=0,000$ ). En consecuencia, se rechazó la hipótesis nula y se aceptó que el perfil ambiental del profesorado fue diferente durante los cuatro momentos del estudio.

- Finalmente, se realizó la prueba t para una muestra con la hipótesis a una sola cola, donde se comparó el promedio de los tres momentos posteriores a la intervención $(320,0)$ con el valor inicial $(227,92)$; evidenciándose diferencias significativas de 42,2 puntos con un intervalo de confianza de 34,0-50,4 ( $\mathrm{t}=10,4 ; \mathrm{p}=0,000)$, con lo que se rechazó la segunda hipótesis nula y se aceptó que el promedio de los tres momentos posteriores a la intervención que fue mayor al del momento inicial.

En definitiva, con las hipótesis probadas se afirma que la intervención comunicativa aplicada mejora el perfil ambiental del profesorado del Colegio Nacional Integrado Jorge Basadre en Santa María del Valle, durante el 2011; es decir, demostró ser efectivo. Queda por confirmar si las dimensiones práctica de los valores y principios ambientales del perfil ambiental pueden ser motivadoras con estrategias educativas, por lo que debería ser investigado en futuros estudios.

En consecuencia, se asume que el profesorado de dicha institución educativa seleccionada cuenta con un perfil ambiental efectivo, y es de esperar un rol proambiental activo, con asimilación y comprensión del conocimiento sobre el ambiente. Su comportamiento, manejo adecuado y racional permitirá la participación responsable y eficaz en la prevención y la solución de los problemas ambientales por parte de la ciudadanía, y contribuirá en mejorar la calidad del ambiente (Barraza, 2000). En suma, el proceso educativo es clave en la formación de convicciones y actitudes éticas, económicas y estéticas, que constituyen la base de una disciplina hacia el ambiente, garantizando su protección, mantenimiento y preservación (Maldonado, 2005). Este hecho, además, indica que los docentes transformarán los comportamientos y actitudes de los estudiantes, de ahí su relevancia como estrategia de protección y cuidado ambiental.

Por lo anterior, se hace necesario reflexionar en la importancia que ejerce el docente en la vida de los individuos, y por tanto el compromiso de hacerlo bien, ya que tiene la oportunidad de formar integralmente a los individuos. Esto transfiriendo una nueva cultura, favoreciendo la continuidad del conocimiento y lo 
que esto representa en todos los aspectos, pero especialmente en este caso en el ambiental, ya que su labor se verá reflejada en el corto plazo en la actitud de los niños, quienes, al ir creciendo en esta educación ambiental continua, se convertirán en profesionistas con una cultura ambiental a favor de un desarrollo sustentable (Luna Tenorio y Fernández, 2005).

En suma, el presente hallazgo resulta efectivo para los propósitos de la educación ambiental, por cuanto los docentes son responsables de los procesos de formación que pueden generar profundos cambios en las dinámicas sociales en las que se encuentran inmersos (Fernández, Porter y Sureda, 2010).

\section{REFERENCIAS BIBLIOGRÁFICAS}

Acebal Expósito, M. (2010). Conciencia Ambiental y Formación de Maestras y Maestros. [Tesis doctoral en Didáctica a la Matemática y Ciencias Experimentales]. Málaga: Servicio de Publicaciones, Universidad de Málaga.

Álvarez, P. y Vega, P. (2009). Actitudes ambientales y conductas sostenibles. Implicaciones para la educación ambiental. Rev. de Psicodidáctica, vol. 14 (2), 245-260.

Barraza, L. (2000). Educar para el futuro: en busca de un nuevo enfoque de investigación en educación ambiental. En Memorias del Foro Nacional de Educación Ambiental (pp. 253-259). México: Universidad Autónoma de Aguascalientes, Secretaría de Educación Pública y Secretaría de Medio Ambiente, Recursos Naturales y Pesca, Aguascalientes.

Bellver Capella, V. (1997). Las ecofilosofías. En J. Ballesteros y J. Pérez Adán. Sociedad y medio ambiente (pp. 251-269). Madrid: Trotta.

Bers Birnes, M. F. Actitud del docente preescolar frente a la educación ambiental. Recuperado el 21 de marzo de 2014. http://www.monografias.com/trabajos14/docentepreesc/ docentepreesc.shtml.

Camarena Gómez, B. O. (2009). Educación Ambiental y Formación de Profesorado: Estudio de Perfiles Ambientales en las Escuelas Normales de Sonora. Tesis doctoral en Pedagogía. Salamanca: Facultad de Ciencias de la Educación, Universidad de Salamanca.

Chocano Zarauz, L. Estado de la educación ambiental en las instituciones educativas del Proyecto Binacional "Bosques de chinchipe". Recuperado el 21 de marzo de 2014. http://www.solucionespracticas.org.pe/bosques/documentos/chinchipe000011.pdf.

Corral Verdugo, V. (2001). Comportamiento Proambiental. Una Introducción al Estudio de las Conductas Protectoras del Ambiente. Santa Cruz de Tenerife, España: RESMA.

Fernández Tarrío, R.; Porter-Bolland, L. y Sureda Negre, J. (2010). Percepciones y conocimientos ambientales de la población infantil y juvenil de una comunidad rural de Veracruz, México. Rev. de Educ. y Des. Ener., 1 (1), 35-43.

Fernández Tarrío, R.; Porter-Bolland, L. y Sureda Negre, J. (2010). Percepciones y conocimientos ambientales de la población infantil y juvenil de una comunidad rural de Veracruz. México. Revista de Educación y Desarrollo, 12. Enero-marzo de 2010. Recuperado el 21 de setiembre de 2014. http://www.cucs.udg.mx/revistas/edu_desarrollo/ anteriores/12/012_Fernandez_Tarrio.pdf.

Fishbein (2011). En Ríos Hernández, I. Comunicación en salud: conceptos y modelos teóricos. Perspectivas de la Comunicación, 4 (1), 123-140. Chile: Universidad de la Frontera Temuco. 
Grob, A. (2005). Meinungen im Umweltbereich und umwelgerechtes Verhalten. Ein psychologisches Ursachennetzmodell. [Tesis doctoral]. Suiza: Universidad de Berna.

Hsu, Roth; González Muñoz y Martín, Grace et al. En Corral Verdugo, V. (2001). Comportamiento Proambiental. Una Introducción al Estudio de las Conductas Protectoras del Ambiente. Santa Cruz de Tenerife, España: RESMA.

López Cózar, P. Formación del profesorado en educación medioambiental. Recuperado el 26 de octubre de 2014. http://www.eduinnova.es/mar2010/medioambiental.pdf.

Luna Tenorio, K. y Fernández Crispín, A. (2005). Educación ambiental en la educación básica del Municipio de Puebla, Puebla (p. 76). Puebla: Benemérita Universidad Autónoma de Puebla. Escuela de Biología.

Maldonado Delgado, H. (2005). La Educación ambiental como herramienta social. Geoenseñanza, 10 (1), 61-67.

McLaughil, A. (1999). El fin del desarrollo. En Cuba Verde. La Habana: Editorial José Martí.

Moràn, G. (2014). Talleres vivenciales: la importancia del ser con el otro. Recuperado el 21 de marzo de 2014. http://www.sht.com.ar/archivo/personal/vivenciales.htm.

Moreno Latorre, E. (2005). La formación inicial en educación ambiental de los profesores de secundaria en periodo formativo. Tesis doctoral. Valencia: Departamento de Didáctica de las Ciencias Experimentales.

Nieda, J. y Macedo, B. (1997). Un currículo científico para estudiantes de 11 a 14 años (p. 41). Madrid: UNESCO-OEI.

Organización Panamericana de la Salud (2011). En Ríos Hernández, I. Comunicación en salud: conceptos y modelos teóricos. Perspectivas de la Comunicación, 4 (1), 123-140. Chile: Universidad de la Frontera Temuco.

Parra Ortiz, J. M. La Educación en valores y su práctica en el aula. Madrid: Universidad Complutense de Madrid. Recuperado el 21 de marzo de 2014. http://www.tendenciaspedagogicas.com/Articulos/2003_08_04.pdf.

Pasco, A.; Villafuerte Armando, V. y Neyra, R. (2010). Influencia del conocimiento técniconormativo de la problemática y la actitud ambiental en la toma de decisiones respecto a la estrategia nacional sobre el cambio climático. Huaraz, 2008. Rev. Aporte Santiaguino, 3 (2), 191-196.

Prochaska, J. O. (1979). Systems of psychotherapy: A transtheoretical analysis. Homewood. Illinois: Dorsey Press.

Sánchez Llanes R. I.; Tamayo M. M.; Barbería Fernández, G.; Soler Rasúa, I. y Soto Soler, D. Estrategia de capacitación sobre educación ambiental para docentes de la filial. Artemisa, Cuba, Ciencias Médicas San Cristóbal. Recuperado el 21 de marzo de 2014. http:// fundacionculturaydeporte.com/pdf/acta_alumn_noheda.pdf.

Sánchez Llanes, R. I.; Tamayo, M. M.; Barbería Fernández, G.; Soler Rasúa, I. y Soto Soler, D. Estrategia de capacitación sobre educación ambiental para docentes de la filial Ciencias Médicas San Cristóbal, Artemisa, Cuba. Recuperado el 21 de marzo de 2014. http://fundacionculturaydeporte.com/pdf/acta_alumn_noheda.pdf. Bers Birnes, M. F. Op. cit., p. 2.

Sandoval Gutiérrez, G. E. El docente de Educación Básica y La Educación Ambiental. Recuperado el 21 de marzo de 2014. http://basica.sep.gob.mx/dgdc/sitio/pdf/inicio/matlinea/2011/FCyE_SEC.pdf.

Servicios Educativos Integrados al Estado de México, Secretaría de Educación de la entidad (2009). Educación Ambiental para la Sustentabilidad. Educación básica. Secundaria. Programa de Estudios 2006 (p. 7). Toluca: Personal académico de la Dirección 
de Educación Secundaria y Servicios de Apoyo. Sta. Cruz Azcapotzaltongo. Edo. Méx. Versión.

Terrón Amigón, E. Educación ambiental y los valores en la educación básica. Un problema de relaciones. Congreso Nacional del medio ambiente. Cumbre del desarrollo sostenible. Comunicación técnica (p. 6). México: Universidad Pedagógica Nacional de México. Recuperado el 23 de agosto de 2014. http://www.conama9.org/conama9/ download/files/CTs/2749_ETerr\%F3n.pdf.

Vega Marcote, P. (2004). La Educación Ambiental en la formación inicial del profesorado. Análisis de un modelo didáctico para el desarrollo de la competencia para la acción a favor del medio. Tesis doctoral en Pedagogía e Didáctica de la Ciencia Experimental. A Coruña: Facultad de Ciencia de la Educación, Universidad de A Coruña.

Zeballos Velarde, M. (2005). Impacto de un Proyecto de Educación Ambiental en Estudiantes de un Colegio en una Zona Marginal de Lima. Tesis para optar el grado de magíster en gerencia social, diciembre (p. 34). Lima: Pontificia Universidad Católica del Perú. 